\title{
COMPARAÇÃO DE DIPIRONA INTRAVENOSA COM METOCLOPRAMIDA INTRAVENOSA NO TRATAMENTO DE CRISE AGUDA DE ENXAQUECA ENSAIO CLÍNICO RANDOMIZADO
}

\author{
Sérgio Murilo Maciel Fernandes Filho' ${ }^{1}$ Márcio Santos Costa², \\ Mariana Torres Fernandes 3 , Miriam Vils Foerster ${ }^{3}$
}

\begin{abstract}
RESUMO - Introdução: A enxaqueca é uma das doenças mais comuns da prática clínica. Objetivo: Comparar o efeito analgésico da dipirona com a metoclopramida no alívio da dor. Método: Conduzimos um estudo piloto randomizado comparando dipirona intravenosa com metoclopramida intravenosa em 27 pacientes apresentando crise de enxaqueca. O objetivo primário foi comparar a redução da intensidade da dor até 2 horas, assim como efeitos colaterais. Resultados: Os grupos tinham faixas etárias semelhantes, mas diferença entre os sexos. Nos homens a metoclopramida reduziu a dor em $80,0 \%$ contra $55,0 \%$ da dipirona $(p=0,052)$. Já nas mulheres houve uma redução de $65,0 \%$ com a metoclopramida e $71,2 \%$ com a dipirona $(p=0,748)$. Esses resultados podem ter sido influenciados pelo reduzido número de pacientes no estudo. Não foram encontrados efeitos colaterais significativos. Conclusão: Não houve diferença quanto à redução da dor nem quanto ao surgimento de efeitos colaterais quando comparados dipirona com metoclopramida.
\end{abstract}

PALAVRAS-CHAVE: dipirona, metoclopramida, enxaqueca.

\begin{abstract}
Comparison of intravenous dipyrone to intravenous metoclopramide in the treatment of acute crisis of migraine: randomized clinical trial

ABSTRACT - Introduction: Migraine is one of the commonest diseases of clinical practice. Objetive: To compare the analgesic effect of dipyrone to metoclopramide in the relief of pain. Method: We conducted a pilot randomized study comparing intravenous dipyrone to intravenous metoclopramide in 27 patients presenting acute crisis of migraine. The primary end point was to compare reduction of intensity of pain up to 2 hours, as well as the development of collateral effects. Results: The groups had similar ages, but there were diffe rence between the sexes. In men, metoclopramide reduced pain in $80.0 \%$ compared with $55.0 \%$ from dipyrone $(p=0.052)$. In women, there was a reduction of $65.0 \%$ with metoclopramide and $71.2 \%$ with dipyrone $(p=0.748)$. The small number of patients in the study might have influenced these results. Significant collateral effects were not found. Conclusion: There was no difference about the intensity of pain reduction neither about the development of collateral effects comparing dipyrone to metoclopramide.
\end{abstract}

KEY WORDS: dipyrone, metoclopramide, migraine.

A enxaqueca (ou migrânea) é uma cefaléia episódica primária caracterizada por alterações neurológicas, autonômicas e gastrointestinais em variadas combinações ${ }^{1}$. Está também entre as doenças menos diagnosticadas e, logo, inadequadamente tratadas da prática clínica, com a maioria dos pacientes nunca tendo procurado um médico ou ter desistido do mesmo ${ }^{2}$. É, entretanto, uma moléstia comum, acometendo com pelo menos um ataque de migrânea por ano, $6 \%$ dos homens e $18 \%$ das mulheres nos Estados
Unidos e até $22 \%$ dos brasileiros ${ }^{1,3}$. Além disso, um dia com migrânea grave tem a classificação máxima da escala de incapacidade neurológica da OMS, sendo equivalente à quadriplegia, demência ou psicose ativa $^{4}$. Apesar disso é uma doença inadequadamente tratada. Enquanto na sua crise aguda a recomendação é de uso de AINES ou paracetamol nos casos leves e derivados de ergotamina ou triptanos nos casos graves ${ }^{1,5}$, há predomínio do uso de opióides nos Estados Unidos e Canadá (o que não é recomendado

Setor de Emergência do Hospital Getúlio Vargas, Recife PE, Brasil (HGV): ${ }^{1}$ Médico, HGV; ${ }^{2}$ Médica, HGV, Mestre em Saúde Materno Infantil pelo Instituto Materno Infantil de Pernambuco; ${ }^{3}$ Acadêmica, Faculdade de Medicina da Universidade Federal de Pemambuco.

Recebido 31 Outubro 2005, recebido na forma final 13 Agosto 2006. Aceito 25 Agosto 2006.

Dr. Sérgio Murilo Maciel Fernandes Filho - Rua Visconde de Itaparica 142 / 104A - 50710-090 Recife PE - Brasil.

E-mail: sergiomurilo@yahoo.com 
por nenhum consenso) ${ }^{6}$. No Brasil, a dipirona tem sido a droga de primeira escolha, principalmente em emergências públicas ${ }^{7,8}$. Apesar de ensaios mostrando sucesso de seu uso ${ }^{9}$, ainda não é uma droga recomendada de primeira linha.

Na busca por uma droga eficaz, de ação específica e poucos efeitos colaterais, ensaios vinham sendo publicado sobre a ação analgésica da metoclopramida na crise aguda da enxaqueca ${ }^{10}$, sendo medicação também usada como anti-emético ${ }^{11}$. Metanálise desses ensaios publicada em 2004 coloca a droga por via p a renteral como eficaz no tratamento da crise ${ }^{12}$, já sendo recomendada por alguns autores como droga de primeira escolha na crise aguda em ambiente hospitalar ${ }^{5}$.

Nosso estudo tem como objetivo comparar a dipirona intravenosa com a metoclopramida intravenosa no tratamento da crise aguda de enxaqueca, avaliando tanto a eficácia analgésica até um período após 2 horas de administração da medicação quanto ao desenvolvimento de efeitos colaterais significativos.

\section{MÉTODO}

Foi realizado um estudo piloto na forma de ensaio clínico randomizado com dois braços ativos: um grupo com dipirona intravenosa e o outro com metoclopramida intravenosa.

O estudo foi realizado no Setor de Emergência do Hospital Getúlio Vargas em Recife, Pernambuco, no período de abril a junho de 2005, tendo sido aprovado por comitê de ética em pesquisa.

Eram convidados a participar do estudo pacientes avaliados na triagem clínica com diagnóstico de cefaléia primária. Como critérios de inclusão, foram adotados os critérios para diagnóstico de enxaqueca da IHS (Intermational Headache Society) (Quadro). Foram excluídos do estudo os pacientes menores de 18 anos ou maiores que 55, com história de crises convulsivas, ou alergia a qualquer das medicações selecionadas.

Após a adequação aos critérios de inclusão e exclusão, o paciente graduava sua dor em escala analógica com dez valores de 1 a 10.
Quadro. Critérios de inclusão, baseados nos critérios diagnós ticos de enxaqueca da IHS (International Headache Society).

Início da dor entre 4 e 72 horas antes do atendimento, sem tratamento ou com tratamento oral sem sucesso

\begin{tabular}{ll}
$\begin{array}{l}\text { Pelo menos } 2 \text { das } 4 \\
\text { caracterísiticas ao lado }\end{array}$ & $\begin{array}{l}\text { Unilateral } \\
\text { Pulsátil } \\
\text { Moderada a grave } \\
\text { Agravada por movimento }\end{array}$ \\
$\begin{array}{l}\text { Presença de pelo menos um } \\
\text { dos sintomas ao lado }\end{array}$ & $\begin{array}{l}\text { Náuseas ou vômitos } \\
\text { Fotofobia } \\
\text { Fonofobia }\end{array}$ \\
\hline
\end{tabular}

Após a avaliação, a seleção para participar no estudo, o primeiro entrevistador se reportava ao pesquisador, que randomizava o paciente para o grupo dipirona ou o grupo metoclopramida. O entrevistador se reportava então ao auxiliar de enfermagem, explicitando a droga selecionada para uso no paciente. Não havia informação acerca da medicação recebida e o paciente via apenas a medicação já diluída em água destilada, tendo ambas as preparações aspecto semelhante.

Após a administração da medicação, um segundo entrevistador, que não tinha ciência da gradação inicial da dor e nem da medicação utilizada, avaliava o paciente até um período máximo de 2 horas após a administração da medicação, graduando novamente sua dor em escala analógica e questionando quanto ao desenvolvimento de efeitos colaterais. Após essa etapa, para a alta hospitalar ou quando do surgimento de nova queixa ou intercorrência, o paciente era reportado novamente ao clínico de plantão.

$\mathrm{Na}$ análise estatística dos dados foi utilizado o teste de Wilcoxon para amostras pareadas a fim de comparar os efeitos de cada um dos tratamentos. Para a comparação entre as drogas avaliamos a variação percentual dos escores de dor, utilizando-se o teste de Mann-Whitney. Em ambas as análises foi adotado o nível de significância de 0,05.

\section{RESULTADOS}

Foram selecionados, através da avaliação pelo clínico de plantão na emergência, como pacientes apresentando cefaléia primária, um total de 31 pacientes.

Tabela 1. Descrição numérica do sexo e da idade dos pacientes, segundo o tratamento administrado.

\begin{tabular}{|c|c|c|c|}
\hline \multirow[b]{2}{*}{ Variável } & \multicolumn{2}{|c|}{ Tratamento } & \multirow[b]{2}{*}{ Total $(n=27)$} \\
\hline & Dipirona $(n=12)$ & Metoclopramida $(n=15)$ & \\
\hline \multicolumn{4}{|l|}{ Sexo } \\
\hline Masculino & $4(33,3 \%)$ & $10(66,7 \%)$ & $14(51,8 \%)$ \\
\hline Feminino & $8(66,7 \%)$ & $5(33,3 \%)$ & $13(48,2 \%)$ \\
\hline \multicolumn{4}{|c|}{ Idade dos homens (anos) } \\
\hline Média (DP) & $32,2(12,6)$ & $29,0(8,6)$ & $29,9(9,5)$ \\
\hline \multicolumn{4}{|c|}{ Idade das mulheres (anos) } \\
\hline Média (DP) & $34,4(9,6)$ & $35,8(6,4)$ & $34,9(8,3)$ \\
\hline
\end{tabular}


Tabela 2. Medianas dos escores de dor, antes e depois de cada tratamento, levando em consideração o sexo dos pacientes.

\begin{tabular}{llccc}
\hline \multirow{2}{*}{ Sexo } & & \multicolumn{2}{c}{ Escore de dor } & \\
\cline { 2 - 3 } & Tratamento & Antes & Depois & P \\
\hline \multirow{2}{*}{ Masculino } & Dipirona & 5,0 & 2,0 & 0,250 \\
& Metoclopramida & 6,5 & 1,0 & 0,002 \\
\multirow{3}{*}{ Feminino } & Dipirona & 7,0 & 2,0 & 0,008 \\
& Metoclopramida & 8,0 & 3,0 & 0,063 \\
\hline
\end{tabular}

Tabela 3. Comparação entre as medianas percentuais dos escores de dor, antes e depois de cada tratamento, levando em consideração o sexo dos pacientes.

\begin{tabular}{lccc}
\hline & \multicolumn{2}{c}{$\begin{array}{c}\text { Mediana da variação percentual dos escores } \\
\text { de dor antes e depois dos tratamentos }\end{array}$} & \\
\cline { 2 - 2 } Variável & Dipirona & Metoclopramida & $\mathrm{p}$ \\
\hline Sexo & & & \\
Masculino & $55,0 \%$ & $80,0 \%$ & 0,052 \\
Feminino & $71,2 \%$ & $65,0 \%$ & 0,748 \\
\hline
\end{tabular}

\begin{tabular}{lcc} 
Tabela 4. Quantidade e descrição dos efeitos colaterais apresentados segundo a droga administrada. \\
\hline Droga & $\begin{array}{c}\text { Quantidade de pacientes que relataram efeitos } \\
\text { colaterais após a administração da droga }\end{array}$ & Descrição \\
\hline $\begin{array}{l}\text { Dipirona } \\
\text { Acometidos (total) }\end{array}$ & $1(12)$ & Diarréia (1) \\
$\begin{array}{l}\text { Metoclopramida } \\
\text { Acometidos (total) }\end{array}$ & $3(15)$ & $\begin{array}{c}\text { Sonolência (2); } \\
\text { Tonturas (1) }\end{array}$ \\
\hline
\end{tabular}

Quatro pacientes foram excluídos do estudo: um menor de 18 anos; um maior de 55 anos; um não preencheu critérios de inclusão e um tinha história de alergia à metoclopramida. Dos 27 pacientes foram randomizados 12 para o grupo com uso de dipirona e 15 para metoclopramida.

Verifica-se que o tratamento metoclopramida foi administrado a $66,7 \%$ dos pacientes do sexo masculino e o tratamento dipirona foi administrado também a $66,7 \%$ dos pacientes do sexo feminino. $A$ idade apresentou um comportamento homogêneo, segundo o sexo e o tratamento (Tabela 1).

De acordo com a avaliação dos escores de dor antes e depois do tratamento, verifica-se que entre os pacientes do sexo masculino apenas tratamento metoclopramida promoveu redução da dor estatisticamente significativa. Entre os pacientes do sexo feminino, foi a dipirona que promoveu redução da dor estatisticamente significante, embora não possamos concluir que não houve redução da dor pro movida pela metoclopramida (Tabela 2).
Entre os pacientes do sexo masculino, observouse que a variação percentual dos escores de dor antes e depois do tratamento foi, em geral, maior nos pacientes tratados com metoclopramida do que nos pacientes tratados com dipirona $(p=0,052)$. Não foi vista diferença entre as pacientes do sexo feminino $(p=$ 0,748 ) (Tabela 3).

Foram observados efeitos colaterais leves em um paciente submetido ao tratamento dipirona (relatou diarréia) em comparação a três pacientes submetidos ao tratamento metoclopramida (dois apresentaram sonolência e um apresentou tontura) (Tabela 4).

\section{DISCUSSÃo}

Este é um estudo piloto randomizado que visou à comparação entre duas drogas de uso rotineiroem emergências nacionais: a dipirona e a metoclopramida, no que concerne ao tratamento da dor na crise aguda de enxaqueca. Dentre os 31 pacientes selecionados como portadores de cefaléia primária pelo atendimento clínico na emergência, 30 foram caracte- 
rizados, após questionamentos sobre os critérios de inclusão e exclusão, como apresentando enxaqueca. Esse total de mais de $90 \%$ de portadores de enxaqueca entre os atendidos na unidade de emergência hospitalar corrobora os valores sugeridos pela literatura, indo de encontro à impressão diagnóstica relatada por estudos com questionários aos médicos, que informam percentuais semelhantes entre a enxaqueca e a cefaléia tensional na procura a unidades hospitalares ${ }^{7}$. Essa ausência de adequação diagnóstica leva ao menor reconhecimento da crise de enxaqueca e, assim, menor procura por tratamento adequado.

A randomização do trabalho apresentou faixas etárias semelhantes entre o grupo submetido à dipirona e o grupo submetido à metoclopramida. No entanto, houve predomínio de mulheres $(66,7 \%)$ nos pacientes submetidos à dipirona, enquanto tivemos o inverso $(66,7 \%$ de homens) no grupo de pacientes submetidos à metoclopramida. Isso leva a que esses grupos devam ser avaliados em separado, visto que há variação entre os sexos ${ }^{13,14}$.

A avaliação individual das drogas mostrou eficácia da metoclopramida nos homens e dipirona nas muIheres $(80,0 \%$ e $71,2 \%$ de redução na dor $p=0,002$ e 0,008 respectivamente), com a metoclopramida em mulheres com $65,0 \%$ de redução na dor $(p=0,063)$. A eficácia não significativa da dipirona quando administrada em homens não está relacionada necessariamente a menor atuação da droga nesse subgrupo devendo-se, provavelmente, ao tamanho da amostra.

Quando da comparação entre os subgrupos não conseguimos determinar se havia diferença entre os tratamentos com dipirona e metoclopramida entre as mulheres, e houve sugestão de superioridade da metoclopramida em relação à dipirona nos homens. Esses dados também devem ser avaliados com cautela, no qual a superioridade encontrada da metoclopramida nos homens pode dever-se a pequena amostragem de pacientes nesse subgrupo submetidos ao tratamento com a dipirona.

No que se refereaos efeitos colaterais houve um maior número nos pacientes submetidos ao tratamento com metoclopramida. Entre os três pacientes que referiram efeitos colaterais com a administração dessa droga, houve uma queixa de tontura e duas de sonolência. É importante lembrar que a sonolência é um efeito esperado na administração da droga e considerado benéfico quando do seu surgimento no paciente com migrânea 5 .

Este estudo é um ensaio piloto e, assim, com deficiências na amostragem e, por conseguinte, em alguns subgrupos após randomização. No entanto, seu desenvolvimento com melhor avaliação dos critérios da IHS para enxaqueca levou a reconhecimento mais adequado dela entre as cefaléias primárias atendidas nessa unidade de emergência hospitalar. Além disso, a análise isolada da eficácia de cada droga, most rou efetividade de ambas, corroborando dados de literatura e, ao menos, ampliando o arsenal terapêutico para o tratamento da crise de dor. No entanto a comparação entre as drogas não mostrou diferença entre as elas nem quanto a sua eficácia (apesar da sugestão de uma maior eficácia da metoclopramida em homens), nem quanto ao surgimento de efeitos colaterais significativos.

Faz-se importante a atenção para o reconhecimento da enxaqueca em emergências, visto que seu tratamento específico é uma possibilidade factível com as descobertas em relação à metoclopramida. Quanto à superioridade da eficácia desta em relação às drogas comumente utilizadas atualmente, em especial a dipirona, um estudo mais amplo e bem controlado deve ser desenvolvido, o que pode levar a mudanças no paradigma do tratamento da enxaqueca no Brasil.

\section{REFERÊNCIAS}

1. Silberstein SD. Migraine. Lancet 2004;363:381-391.

2. Ferrari MD. Migraine. Lancet 1998;351:1043-1052.

3. Queiroz LP. Um estudo epidemiológico de cefaléia no Distrito Sede do Município de Florianópolis, SC. Migrâneas e Cefaléias, 2002,5(1).

4. Menken M, Munsat TL, Toole, JF. The global burden of disease study: implications for neurology. Arch Neurol 2000;57:418-420.

5. Bajwa DW, Sabahat A. Acute and preventive treatment of migraine headache in adults. In Rose BD (ed). Up to date. Wellesley: UpTo Date, 2005.

6. Vinson DR. Treatment patterns of isolated benign headache in US department emergency departments. Ann Emerg Med 2002;39:215-222.

7. Fragoso YD, Fonseca PL, Fortinguerra MB, et al. Management of primary headache in emergency services of Santos and sorrounding towns. S Paulo Med J 1998;116:1650-1653.

8. Bigal ME, Bordini CA, Speciali JG. Tratamento da cefaléia em uma unidade de emergência da cidade de Ribeirão Preto. Arq Neuropsiquiatr 1999;57:813-819.

9. Bigal ME, Bordini CA, Tepper SJ, Speciali JG. Intravenous dipyrone in the acute treatment of migraine without aura and migraine with aura: a randomized, double blind, placebo controlled study. Headache 2002;42:862-871.

10. Schwarzberg MN. Application of metoclopramide specificity in migraine attacks therapy. Headache 1994;34:439-441.

11. Pe routka SJ. Drugs effective in the therapy of migraine. In Goodman \& Gilman. The pharmacological basis of therapeutics, $9^{\text {th }}$ ed. New York: McGraw-Hill, 1996:487-502.

12. Colman I, Brown MD, Innes GD, Grafstein E, Roberts TE, Rowe BH. $\mathrm{P}$ a renteral metoclopramide for acute migraine: meta-analysis of randomised controlled trials. BMJ 2004;329:1369-1373.

13. Couturier EG, Bomhof MA, Neven AK, van Dujin NP. Menstrual migraine in a representative Dutch population sample: prevalence, disability and treatment. Cephalalgia 2003;23:302-308.

14. MacGregor EA, Hackshaw A. Prevalence of migraine on each day of the natural menstrual cycle. Neurology 2004;63:351-353. 